NONCOMMUTATIVE GEOMETRY

AND QUANTUM GROUPS

BANACH CENTER PUBLICATIONS, VOLUME 61

INSTITUTE OF MATHEMATICS

POLISH ACADEMY OF SCIENCES

WARSZAWA 2003

\title{
HOPF-TYPE CYCLIC COHOMOLOGY VIA THE KAROUBI OPERATOR
}

\author{
GEORGIY I. SHARYGIN \\ Group of Mathematics, ITEP \\ B. Cheremushkinskaia ul., 25, 117218 Moscow, Russia \\ E-mail: sharygin@gate.itep.ru
}

\begin{abstract}
We employ the notion of the universal differential calculus to propose yet another approach to the Hopf-type cohomology of Hopf algebras.
\end{abstract}

1. Introduction. In their papers (see $[3,4,5]$ ), A. Connes and H. Moscovici provided the explicit structure of a cocyclic module defining the so-called Hopf-type cyclic cohomology of a Hopf algebra. Later on, M. Crainic showed that this cocyclic module could be obtained as the space of coinvariants of a Hopf-algebra action on some other cyclic module. Some further generalizations and developments were made in the papers [23], [1], [15], and most recent results in this field can be found, for instance, in the papers $[13,14]$ and $[16]$.

The purpose of this paper is to describe the Hopf-type cohomology of a Hopf algebra in terms of a subcomodule of the so-called algebra of non-commutative differential forms associated with the Hopf algebra. It turns out that to any modular pair in involution $(\delta, \sigma)$ one can associate a subcomplex of this differential algebra that is stable under the Karoubi operator $\kappa$ (see papers [7, 8] and [17]) or its twisted version $\kappa_{\xi}$ (see (75) and [19]).

In addition to giving a new point of view on this cohomology theory, this approach seems to have some virtues of its own. For instance, one can try to define some similar sort of cyclic homology when a more general object is used instead of a modular pair. Besides this, it can be used to establish bridges between cyclic cohomology and Hopf-Galois theory, as developed in papers of T. Brzeziński, M. Đurđevič, P. M. Hajac, S. Majid $[18,9,10,11,2,12]$ and others.

Let us first of all recall the construction of Hopf-type cohomology due to A. Connes and H. Moscovici. Here and below $\mathcal{H}$ will denote a Hopf algebra over a field of characteristic 0 (mostly $\mathbb{C}$ ). Let $m, \Delta, 1, \epsilon$ and $S$ be the multiplication, comultiplication (or

2000 Mathematics Subject Classification: Primary 58B34.

The paper is in final form and no version of it will be published elsewhere.

The author was partially supported by the grant RFFI 01-01-0546. 
diagonal), unit, counit and antipode of $\mathcal{H}$, respectively. Below we shall usually skip $m$ in our formulas, and use the standard (Sweedler's, see [22]) notation for the diagonal (coproduct):

$$
\Delta(h)=h_{(1)} \otimes h_{(2)} \quad(\text { summation understood }) .
$$

Let $\sigma$ be a group-like element in $\mathcal{H}, \delta: \mathcal{H} \rightarrow \mathbb{C}$ an algebraic character, and $\delta(\sigma)=1$. One says that $(\delta, \sigma)$ is a modular pair in involution if

$$
S_{\delta}^{2}(h)=\sigma h \sigma^{-1}, \forall h \in \mathcal{H}, \quad \text { where } \quad S_{\delta}(h):=\delta\left(h_{(1)}\right) S\left(h_{(2)}\right) .
$$

This is equivalent to the equation $\left(\sigma^{-1} S_{\delta}\right)^{2}=1$.

Given a modular pair in involution, one defines a (co)cyclic module $\mathcal{H}_{(\delta, \sigma)}^{\sharp}$ as follows. (Recall that the cyclic category is self-dual, so that it is not necessary to pay much attention to distinguishing cyclic and cocyclic objects.) The objects are $\left(\mathcal{H}_{(\delta, \sigma)}^{\sharp}\right)_{n}=\mathcal{H}^{\otimes n}$, $n \in \mathbb{N}$, and structure maps (coface, codegeneracy and cyclic permutation operators)

$$
\begin{aligned}
\delta_{i}: \mathcal{H}_{n}^{\sharp} \rightarrow \mathcal{H}_{n+1}^{\sharp}, \quad i=0, \ldots, n+1, \\
\sigma_{i}: \mathcal{H}_{n}^{\sharp} \rightarrow \mathcal{H}_{n-1}^{\sharp}, \quad i=1, \ldots, n, \\
\tau_{n}: \mathcal{H}_{n}^{\sharp} \rightarrow \mathcal{H}_{n}^{\sharp},
\end{aligned}
$$

are given by

$$
\begin{aligned}
& \delta_{i}\left(h_{1}, \ldots, h_{n}\right)= \begin{cases}\left(1, h_{1}, \ldots, h_{n}\right), & i=0 \\
\left(h_{1}, \ldots, \Delta\left(h_{i}\right), \ldots, h_{n}\right), & 1 \leq i \leq n, \\
\left(h_{1}, \ldots, h_{n}, \sigma\right), & i=n+1,\end{cases} \\
& \sigma_{i}\left(h_{1}, \ldots, h_{n}\right)=\epsilon\left(h_{i}\right)\left(h_{1}, \ldots, h_{i-1}, \hat{h}_{i}, h_{i+1}, \ldots, h_{n}\right), 1 \leq i \leq n \text {, } \\
& \tau_{n}\left(h_{1}, h_{2}, \ldots, h_{n}\right)=S_{\delta}\left(h_{1}\right) \cdot\left(h_{2}, \ldots, h_{n}, \sigma\right) .
\end{aligned}
$$

Here in the last formula we assume that $\mathcal{H}$ acts on its own tensor power as follows:

$$
h \cdot\left(h_{1}, \ldots, h_{n}\right)=\left(h_{(1)} h_{1}, \ldots, h_{(n)} h_{n}\right) .
$$

For any cocyclic module one can define its cyclic, negative cyclic and periodic cyclic cohomology. To this end one has to consider the cyclic, negative and periodic complexes, respectively. (See, for example, the book of Loday [20] and [8].) For instance, periodic cohomology is defined by the following super-complex:

$$
C P_{i}=\bigoplus_{n \equiv i(\bmod 2)} \mathcal{H}_{n}^{\sharp}, i=0,1,
$$

equipped with differentials $b: \mathcal{H}_{n}^{\sharp} \rightarrow \mathcal{H}_{n+1}^{\sharp}$ (Hochschild operator) and $B: \mathcal{H}_{n}^{\sharp} \rightarrow \mathcal{H}_{n-1}^{\sharp}$, defined as follows:

$$
\begin{aligned}
b & =\sum_{i=0}^{n+1}(-1)^{i} \delta_{i}, \\
B & =-N \circ \tilde{\sigma}_{0} \circ\left(1-\tau_{n}\right),
\end{aligned}
$$

where

$$
\begin{aligned}
& N=\sum_{i=0}^{n-1}(-1)^{i(n-1)} \tau_{n-1}^{i}, \\
& \tilde{\sigma}_{0}=\sigma_{n} \circ \tau_{n} .
\end{aligned}
$$


Recall that we deal with a cocyclic module here, so that the usual formula for the differential $B$ in the mixed complex associated with a cyclic module is inverted. Also that is the reason why we use direct sum rather than the direct product of homogeneous components in $(8)$.

2. Special case: $(\delta, \sigma)=(\epsilon, 1)$. Let $\mathcal{H}$ be a Hopf algebra. We shall denote by $\Omega(\mathcal{H})$ the universal unital differential graded algebra, generated by $\mathcal{H}$. Recall that

$$
\begin{gathered}
\Omega(\mathcal{H})=\bigoplus_{n \geq 0} \Omega_{n}(\mathcal{H}), \\
\Omega_{0}(\mathcal{H})=\mathcal{H}, \quad \Omega_{1}(\mathcal{H})=\operatorname{ker}(m: \mathcal{H} \otimes \mathcal{H} \rightarrow \mathcal{H}), \\
\Omega_{n}(\mathcal{H})=\underbrace{\Omega_{1}(\mathcal{H}) \otimes_{\mathcal{H}} \Omega_{1}(\mathcal{H}) \otimes_{\mathcal{H}} \ldots \otimes_{\mathcal{H}} \Omega_{1}(\mathcal{H})}_{n} .
\end{gathered}
$$

The differential $d: \mathcal{H} \rightarrow \Omega_{1}(\mathcal{H})$ is given by

$$
d(x)=1 \otimes x-x \otimes 1 .
$$

One can prove that any element $\theta$ in $\Omega_{n}(\mathcal{H})$ can in a unique way be written in the form

$$
\theta=\sum_{i} a_{0}^{i} d a_{1}^{i} d a_{2}^{i} \ldots d a_{n}^{i}, \quad a_{j}^{i} \in \mathcal{H}
$$

Now it is clear that

$$
d \theta=\sum_{i} d a_{0}^{i} d a_{1}^{i} d a_{2}^{i} \ldots d a_{n}^{i}
$$

So far, the coalgebra structure has not yet come to the scene. One can define the universal differential algebra associated to any unital algebra $\mathcal{A}$ in precisely the same way. But now, since $\mathcal{H}$ is a Hopf algebra, one can also define left and right coactions of $\mathcal{H}$ on $\Omega(\mathcal{H})$. Put

$$
\Delta_{R}(\theta)=\sum_{i} a_{0,(1)}^{i} d a_{1,(1)}^{i} d a_{2,(1)}^{i} \ldots d a_{n,(1)}^{i} \otimes a_{0,(2)}^{i} a_{1,(2)}^{i} a_{2,(2)}^{i} \ldots a_{n,(2)}^{i},
$$

and

$$
\Delta_{L}(\theta)=\sum_{i} a_{0,(1)}^{i} a_{1,(1)}^{i} a_{2,(1)}^{i} \ldots a_{n,(1)}^{i} \otimes a_{0,(2)}^{i} d a_{1,(2)}^{i} d a_{2,(2)}^{i} \ldots d a_{n,(2)}^{i}
$$

The fact that these formulae really determine well-defined maps follows from the universal properties of $\Omega(\mathcal{H})$. Moreover, (17) and (18) define the right- and left-Hopf-comodule algebra structures on $\Omega(\mathcal{H})$. That is, the map $\Delta_{R}: \Omega(\mathcal{H}) \rightarrow \Omega(\mathcal{H}) \otimes \mathcal{H}$ is an algebra morphism, and so is $\Delta_{L}$. In particular, $\Omega(\mathcal{H})$ is a left and right Hopf module over the Hopf algebra $\mathcal{H}=\Omega_{0}(\mathcal{H})$. Recall that a right module $M$ over a Hopf algebra $\mathcal{H}$ is called a (right) Hopf module if it is equipped with a coaction $\Delta_{M}: M \rightarrow M \otimes \mathcal{H}, m \mapsto m_{\langle 0\rangle} \otimes m_{\langle 1\rangle}$, such that $(m h)_{\langle 0\rangle} \otimes(m h)_{\langle 1\rangle}=m_{\langle 0\rangle} h_{(1)} \otimes m_{\langle 1\rangle} h_{(2)}$ for all $m \in M, h \in \mathcal{H}$.

In general, conditions that the formulas (17) and (18) define such structures impose additional restrictions on the structure of a differential calculus $\Omega^{\prime}(\mathcal{H})$. Differential calculi verifying these restrictions are called bicovariant. This matter is accurately explained, 
e.g., in [24] (see also [9]), where the general definition of a bicovariant differential calculus on a Hopf algebra is given. ${ }^{1}$

Observe (see (16)) that both maps (17) and (18) preserve the differential. Hence, in particular, the subspaces of left and right coinvariants are differential graded subalgebras in $\Omega(\mathcal{H})$. Let us describe explicitly the structure of these subalgebras. For instance, let us take the space of right coinvariants $\Omega(\mathcal{H})^{\text {co } \mathcal{H}} \stackrel{\text { def }}{=} \Omega^{R}(\mathcal{H})$. (The symbol $\Omega_{n}^{R}(\mathcal{H})$ will denote the degree $n$ homogeneous component of this subalgebra).

In the general theory of Hopf modules (e.g., see [22]), it is proven that any (right) Hopf module $M$ over $\mathcal{H}$ is isomorphic (as a Hopf module) to the "free" Hopf module $M^{\text {co } \mathcal{H}} \otimes \mathcal{H}$. The isomorphism $M^{\text {co } \mathcal{H}} \otimes \mathcal{H} \rightarrow M$ sends $m \otimes h \in M^{\text {co } \mathcal{H}} \otimes \mathcal{H}$ to $m h \in M$, and its inverse sends $m$ to $m_{\langle 0\rangle} S\left(m_{\langle 1\rangle}\right) \otimes m_{\langle 2\rangle}$. A consequence of this fact is (see [24] and [9], App. A) the following isomorphism of right $\mathcal{H}$-Hopf modules:

$$
\Omega_{n}(\mathcal{H}) \cong \Omega_{n}^{R}(\mathcal{H}) \otimes \mathcal{H}, \quad \forall n \in \mathbb{N} .
$$

Moreover, one can show ([9], App. A) that the map

$$
\pi^{R}: \mathcal{H} \rightarrow \Omega_{1}^{R}(\mathcal{H}), \quad h \mapsto d h_{(1)} \cdot S\left(h_{(2)}\right)
$$

maps isomorphically the kernel of the counit of $\mathcal{H}$ on the space $\Omega_{1}^{R}(\mathcal{H})$. This isomorphism can be extended to all degrees (this can be shown, e.g., with the help of the formula (24) below, see also [9], App. A):

$$
\Omega_{n}^{R}(\mathcal{H}) \cong \Omega_{1}^{R}(\mathcal{H})^{\otimes n} \cong(\operatorname{ker} \epsilon)^{\otimes n} .
$$

One can compute the differential and the left $\mathcal{H}$-action and coaction on $\Omega^{R}(\mathcal{H})$ in the terms of the map $\pi^{R}$ :

$$
\begin{gathered}
d h=d h_{(1)} \cdot S\left(h_{(2)}\right) h_{(3)}=\pi^{R}\left(h_{(1)}\right) h_{(2)}, \\
d \pi^{R}(h)=-d h_{(1)} d S\left(h_{(2)}\right)=d h_{(1)} S\left(h_{(2)}\right) d h_{(3)} S\left(h_{(4)}\right)=\pi^{R}\left(h_{(1)}\right) \pi^{R}\left(h_{(2)}\right), \\
a \cdot \pi^{R}(h)=a_{(1)} \pi^{R}(h) S\left(a_{(2)}\right) a_{(3)}=\pi^{R}\left(a_{(1)} h-\epsilon(h) a_{(1)}\right) a_{(2)}, \\
\Delta_{L}\left(\pi^{R}(h)\right)=h_{(1)} S\left(h_{(3)}\right) \otimes \pi^{R}\left(h_{(2)}\right)
\end{gathered}
$$

(for all $a, h \in \mathcal{H}$ ). Therefore, in the terms of the isomorphisms (19) and (21), one can write the differential and left action of $\mathcal{H}$ on $\Omega(\mathcal{H})$ as follows:

$$
\begin{gathered}
d h \leftrightarrow \pi^{R}\left(h_{(1)}\right) \otimes h_{(2)}, \\
d \pi^{R}(h) \leftrightarrow \pi^{R}\left(h_{(1)}\right) \otimes \pi^{R}\left(h_{(2)}\right), \\
a \cdot \pi^{R}(h) \leftrightarrow \pi^{R}\left(a_{(1)} h-\epsilon(h) a_{(1)}\right) \otimes a_{(2)} .
\end{gathered}
$$

Here we consider the case $n=1$ in (19), formulas for other degrees follow from this case in an obvious way. In particular, taking $h$ in $\operatorname{ker} \epsilon$ in the formula (28), we obtain the following description of the left action of $\mathcal{H}$ on the bimodule $\Omega_{1}(\mathcal{H})$, which we identify with ker $\epsilon \otimes \mathcal{H}$ with the help of the composite map $\operatorname{ker} \epsilon \otimes \mathcal{H} \stackrel{\pi^{R} \otimes \text { id }}{\longrightarrow} \Omega_{1}^{R}(\mathcal{H}) \otimes \mathcal{H} \rightarrow \Omega_{1}(\mathcal{H}), k \otimes h \mapsto \pi^{R}(k) h$ :

$$
a \cdot(k \otimes h)=a_{(1)} k \otimes a_{(2)} h .
$$

\footnotetext{
${ }^{1}$ One should however keep in mind that in the cited paper the term "differential calculus" is used to denote only the first-order part of an algebra of differential forms.
} 
This formula extends in a natural way to the $n$-th degree component of $\Omega(\mathcal{H}), \Omega_{n}(\mathcal{H}) \cong$ $(\operatorname{ker} \epsilon)^{\otimes n} \otimes \mathcal{H}$. The isomorphism is given by

$$
h_{1} \otimes \ldots \otimes h_{n} \otimes h \mapsto \pi^{R}\left(h_{1}\right) \ldots \pi^{R}\left(h_{n}\right) h, h_{1}, \ldots, h_{n} \in \operatorname{ker} \epsilon, h \in \mathcal{H} .
$$

We get for all $a \in \mathcal{H}$

$$
a \cdot\left(h_{1} \otimes h_{2} \otimes \ldots \otimes h_{n} \otimes h\right)=a_{(1)} h_{1} \otimes a_{(2)} h_{2} \otimes \ldots \otimes a_{(n)} h_{n} \otimes a_{(n+1)} h .
$$

Recall that one can define on $\Omega(\mathcal{H})$ the Hochschild differential $b$ and the Karoubi operator $\kappa$ in the following way (see [8], §3):

$$
b(\omega d a)=(-1)^{|\omega|}(\omega a-a \omega),
$$

where $a \in \mathcal{H}, \omega \in \Omega(\mathcal{H})$, and

$$
\kappa=1-b d-d b
$$

Explicitly, one can show that

$$
\kappa(\omega d a)=(-1)^{|\omega|} d a \omega .
$$

For our purposes it would be useful to consider a little bit different operators, $b^{\prime}, \kappa^{\prime}$ :

$$
\begin{aligned}
b^{\prime}(d a \omega) & =a \omega-\omega a, \\
\kappa^{\prime}(d a \omega) & =b^{\prime} d+d b^{\prime}-1 .
\end{aligned}
$$

Explicitly, we have

$$
\kappa^{\prime}(d a \omega)=(-1)^{|\omega|} \omega d a .
$$

Let $B^{\prime}=\sum_{i=0}^{n}{\kappa^{\prime}}^{i} \circ d$ on $\Omega_{n}(\mathcal{H})$. Then $B^{\prime}$ is analogous to the operator $B$ from [8], and the primed operators $b^{\prime}, \kappa^{\prime}$ and $B^{\prime}$ have all the usual properties of $b, \kappa$ and $B$, see $\S 3$ of [8]. This can be proven by a slight modification of the reasoning used in the quoted paper for the unprimed operators. Hence, we conclude that $b^{\prime}$ and $B^{\prime}$ induce a mixed complex structure on $\Omega(\mathcal{H})$. Moreover, the formulas (34) and (36) show that

$$
\kappa^{\prime}=\kappa^{-1}
$$

and

$$
b^{\prime}=-b \circ \kappa^{\prime}
$$

The following theorem is the main result of this section.

TheOREM 1. Let $\mathcal{H}$ be a Hopf algebra and the modular pair $(1, \epsilon)$ be such that the conditions of [4] are satisfied (i.e. $S^{2}=1$ ). Then $\Omega^{R}(\mathcal{H})$ is a differential graded subalgebra of $\Omega(\mathcal{H})$ which is stable under the Hochschild differential b and Karoubi operator $\kappa$ (or equivalently under $b^{\prime}$ and $\left.\kappa^{\prime}\right)$, and hence is a mixed subcomplex of $(\Omega(\mathcal{H}), b, B)$. The same is true about $\Omega^{L}(\mathcal{H})$. Moreover, the periodic homology of $\Omega^{R}(\mathcal{H})$ with the mixed complex structure induced from $\left(\Omega(\mathcal{H}), b^{\prime}, B^{\prime}\right)$ is naturally isomorphic to the periodic Hopf-type cohomology $H P_{\epsilon, 1}^{*}(\mathcal{H})$ of the Hopf algebra $\mathcal{H}$.

REMARK 1. It is important to observe that though the mixed complex structure in $\Omega^{R}(\mathcal{H})$ is induced from $\Omega(\mathcal{H})$, it is not quite correct to consider the periodic cohomology complex of $\Omega^{R}(\mathcal{H})$ as a subcomplex of the Cuntz and Quillen periodic complex for 
$\Omega(\mathcal{H})$, as defined in [8]. In effect, except for the difference in the cyclic structure (see formulas (37) and (38)), the latter is formed with the help of the direct product of homogeneous components (see the cited paper), while the former deals with their direct sum (see $(8))$.

Proof. The fact that $\Omega^{R}(\mathcal{H})$ (and $\Omega^{L}(\mathcal{H})$ as well) is a differential graded subalgebra of $\Omega(\mathcal{H})$ follows directly from the discussion above. Now we shall prove the second statement of this theorem, i.e. that these subalgebras are closed under the Hochschild and Karoubi operators. We shall confine our attention to $\Omega^{R}(\mathcal{H})$. (The case of $\Omega^{L}(\mathcal{H})$ can be treated similarly.)

First of all, let us note that it is enough to prove the stability of $\Omega^{R}(\mathcal{H})$ only under the action of the primed operators $b^{\prime}$ and $\kappa^{\prime}$. (This follows from the formulas (37) and (38).) Therefore, let us start with showing that $\Omega^{R}(\mathcal{H})$ is stable under $b^{\prime}$. To this end, we shall directly compute the image of an element $\omega \in \Omega^{R}(\mathcal{H})$ under $b^{\prime}$. First, let $\omega$ belong to $\Omega_{1}^{R}(\mathcal{H})$. We compute:

$$
\begin{aligned}
b^{\prime}(\omega) & =b^{\prime}\left(d a_{(1)} S\left(a_{(2)}\right)\right)=a_{(1)} S\left(a_{(2)}\right)-S\left(a_{(2)}\right) a_{(1)} \\
& =\epsilon(a) \cdot 1-S\left(a_{(2)}\right) S^{2}\left(a_{(1)}\right)=\epsilon(a) \cdot 1-S\left(S\left(a_{(1)}\right) a_{(2)}\right) \\
& =\epsilon(a) \cdot 1-\epsilon(a) \cdot 1=0,
\end{aligned}
$$

which is, of course, a right-coinvariant element. Here we have used the formula (34) and the possibility to represent any element in $\Omega_{1}^{R}(\mathcal{H})$ as the image of some $a \in \mathcal{H}$ under the map $\pi^{R}$ from (20).

Now, if the element $\omega$ belongs to $\Omega_{n}^{R}(\mathcal{H}) \cong \Omega_{1}^{R}(\mathcal{H})^{\otimes n} \cong \operatorname{ker} \epsilon^{\otimes n}$ (see $(20)$ ) we use the formulas (24) and (30) to compute:

$$
\begin{aligned}
b^{\prime}(\omega) & =b^{\prime}\left(\pi^{R}\left(a_{1}\right) \pi^{R}\left(a_{2}\right) \ldots \pi^{R}\left(a_{n}\right)\right)=b^{\prime}\left(d\left(a_{1,(1)}\right) S\left(a_{1,(2)}\right) \omega^{\prime}\right) \\
& =a_{1,(1)} S\left(a_{1,(2)}\right) \omega^{\prime}-S\left(a_{1,(2)}\right) \omega^{\prime} a_{1,(1)} \\
& =-\left(\pi^{R}\left(S\left(a_{1,(n+1)}\right) a_{2}\right) \ldots \pi^{R}\left(S\left(a_{1,(3)}\right) a_{n}\right)\right)\left(S\left(a_{1,(2)}\right) a_{1,(1)}\right) \\
& =-\left(\pi^{R}\left(S\left(a_{1,(n+1)}\right) a_{2}\right) \ldots \pi^{R}\left(S\left(a_{1,(3)}\right) a_{n}\right)\right)\left(S\left(a_{1,(2)}\right) S^{2}\left(a_{1,(1)}\right)\right) \\
& =-\left(\pi^{R}\left(S\left(a_{1,(n+1)}\right) a_{2}\right) \ldots \pi^{R}\left(S\left(a_{1,(3)}\right) a_{n}\right)\right)\left(S\left(S\left(a_{1,(1)}\right) a_{1,(2)}\right)\right) \\
& =-\pi^{R}\left(S\left(a_{1,(n-1)}\right) a_{2}\right) \pi^{R}\left(S\left(a_{1,(n-2)}\right) a_{3}\right) \ldots \pi^{R}\left(S\left(a_{1,(1)}\right) a_{n}\right) .
\end{aligned}
$$

Here we suppose that $a_{i} \in \operatorname{ker} \epsilon, i=1, \ldots, n$ (see (30)), and in order to abbreviate our notation we put $\omega^{\prime}=\pi^{R}\left(a_{2}\right) \pi^{R}\left(a_{3}\right) \ldots \pi^{R}\left(a_{n}\right)$. Clearly, $b^{\prime}(\omega)$ lies in $\Omega_{n-1}^{R}(\mathcal{H})$. One should view this computation as carried out in the first of the isomorphic spaces listed in (21), although we have implicitly used all the isomorphisms of this formula.

Of course, since the inverse of the Karoubi operator $\kappa^{\prime}$ is written in terms of $d$ and $b^{\prime}$ (see $(35)$ ), one can conclude that $\Omega^{R}(\mathcal{H})$ is stable under its action. This proves the second statement of our theorem.

However, below we shall need an explicit formula for this operator written in terms of the isomorphism (20). Therefore, we use (36) and, employing the notation introduced in the paragraph following (35) and (40), compute: 


$$
\begin{aligned}
\kappa^{\prime}\left(d a_{1,(1)}\right. & \left.S\left(a_{1,(2)}\right) \omega^{\prime}\right)=(-1)^{\left|\omega^{\prime}\right|} S\left(a_{1,(2)}\right) \omega^{\prime} d a_{1,(1)} \\
& =(-1)^{\left|\omega^{\prime}\right|}\left(\pi^{R}\left(S\left(a_{1,(n+1)}\right) a_{2}\right) \ldots \pi^{R}\left(S\left(a_{1,(3)}\right) a_{n}\right)\right)\left(S\left(a_{1,(2)}\right) d a_{1,(1)}\right) \\
& =(-1)^{\left|\omega^{\prime}\right|}\left(\pi^{R}\left(S\left(a_{1,(n+1)}\right) a_{2}\right) \ldots \pi^{R}\left(S\left(a_{1,(3)}\right) a_{n}\right)\right)\left(S\left(a_{1,(2)}\right) d S^{2}\left(a_{1,(1)}\right)\right) \\
& =(-1)^{\left|\omega^{\prime}\right|+1}\left(\pi^{R}\left(S\left(a_{1,(n)}\right) a_{2}\right) \ldots \pi^{R}\left(S\left(a_{1,(2)}\right) a_{n}\right)\right) \pi^{R}\left(S\left(a_{1,(1)}\right)\right) \\
& =(-1)^{n} \pi^{R}\left(S\left(a_{1,(n)}\right) a_{2}\right) \ldots \pi^{R}\left(S\left(a_{1,(2)}\right) a_{n}\right) \pi^{R}\left(S\left(a_{1,(1)}\right)-\epsilon\left(a_{1,(1)}\right)\right) .
\end{aligned}
$$

Here in the third line we used the assumption that $S^{2}=$ id. In other words, this can be written as

$$
\begin{aligned}
\kappa^{\prime}: \operatorname{ker} \epsilon^{\otimes n} & \rightarrow \operatorname{ker} \epsilon^{\otimes n}, \\
\left(h_{1}, h_{2}, \ldots, h_{n}\right) & \mapsto(-1)^{n} \operatorname{proj}^{\prime}\left(S\left(a_{1}\right) \cdot\left(h_{2}, \ldots, h_{n}, 1\right)\right) .
\end{aligned}
$$

Here proj' denotes the standard projection $\operatorname{proj}^{\prime}: \mathcal{H}^{\otimes n} \rightarrow \operatorname{ker} \epsilon^{\otimes n}$ that sends each component $h_{i}$ to $h_{i}-\epsilon\left(h_{i}\right)$.

The similarity between these formulae and the structure of the cyclic module introduced by A. Connes and H. Moscovici is conspicuous. For instance, the cyclic operator $\tau_{n}$ of this module is given by

$$
\tau\left(h_{1}, h_{2}, \ldots, h_{n}\right)=S\left(h_{1}\right)\left(h_{2}, \ldots, h_{n}, 1\right),
$$

that is, it coincides with $\kappa^{\prime}$, up to the sign and projection on the kernel of the counit.

In view of this observation, let us finally show that the cohomology of the induced mixed subcomplex $\left(\Omega^{R}(\mathcal{H}), b^{\prime}, B^{\prime}\right)$ coincides with the Hopf-type cohomology of Connes and Moscovici.

To this end, we first consider the cocyclic object $\mathcal{H}_{(\epsilon, 1)}^{\sharp}$ defined in [4] and [6], see Section 1 above. Recall that the mixed complex associated with $\mathcal{H}_{(\epsilon, 1)}^{\sharp}$ is defined by the operators $\tilde{b}=\sum_{i=0}^{n+1}(-1)^{i} \delta_{i}$, where

$$
\delta_{i}\left(h_{1}, h_{2}, \ldots, h_{n}\right)= \begin{cases}\left(1, h_{1}, h_{2}, \ldots, h_{n}\right), & i=0, \\ \left(h_{1}, \ldots, \Delta\left(h_{i}\right), \ldots, h_{n}\right), & 1 \leq i \leq n \\ \left(h_{1}, h_{2}, \ldots, h_{n}, 1\right), & i=n+1\end{cases}
$$

and $\tilde{B}=-N \circ \tilde{\sigma}_{0} \circ\left(1-\tau_{n}\right)$, where

$$
\tilde{\sigma}_{0}\left(h_{1}, h_{2}, \ldots, h_{n}\right)=S\left(h_{1}\right) \cdot\left(h_{2}, \ldots, h_{n}\right),
$$

and $N$ is given by the formula (11). This is a special case of the formulas (5)-(7). Here we use tilde to distinguish these maps from the Cuntz-Quillen operators on $\Omega(\mathcal{H})$ introduced above.

Now, consider the subcomplex $\left(\tilde{\mathcal{H}}_{(\epsilon, 1)}^{\sharp}, \tilde{b}^{\prime}, \tilde{B}^{\prime}\right)$ of this mixed complex: $\left(\tilde{\mathcal{H}}_{(\epsilon, 1)}^{\sharp}\right)_{n}=$ $\operatorname{ker} \epsilon^{\otimes n}=\bigcap_{i=1}^{n} \operatorname{ker} \sigma_{i}, i \geq 1$ and $\left(\tilde{\mathcal{H}}_{(\epsilon, 1)}^{\sharp}\right)_{0}=\mathbb{C}$. It follows from the (co)cyclic module relations, satisfied by the maps $\delta_{i}, \sigma_{j}$ and $\tau_{n}$ (see Loday's book [20]) that both $\tilde{b}$ and $\tilde{B}$ map this subspace to itself. (We will denote by $\tilde{b}^{\prime}$ and $\tilde{B}^{\prime}$ the restrictions to $\tilde{\mathcal{H}}_{(\epsilon, 1)}^{\sharp}$ of $\tilde{b}$ and $\tilde{B}$, respectively.)

Lemma 2. The natural inclusion of $\tilde{\mathcal{H}}_{(\epsilon, 1)}^{\sharp}$ into $\mathcal{H}_{(\epsilon, 1)}^{\sharp}$ induces an isomorphism on cyclic cohomology. 
Proof. This is a direct consequence of the fact that this inclusion yields an isomorphism of the Hochschild homologies of these two complexes (i.e. their homologies with respect to the differentials $\tilde{b}$ and $\tilde{b}^{\prime}$ ), which is a standard fact of homological algebra. (In fact, $\tilde{\mathcal{H}}_{(\epsilon, 1)}^{\sharp}$ is just the normalization of $H_{(\epsilon, 1)}^{\sharp}$ with respect to the codegeneracy operators $\sigma_{i}$.)

Now, as we have observed above, the homomorphism

$$
\mathcal{H}^{\otimes n} \ni h_{1} \otimes h_{2} \otimes \ldots \otimes h_{n} \stackrel{\left(\pi^{R}\right)}{\mapsto}{ }^{\otimes}-\pi^{R}\left(h_{1}\right) \pi^{R}\left(h_{2}\right) \ldots \pi^{R}\left(h_{n}\right) \in \Omega_{n}^{R}(\mathcal{H}),
$$

induces an isomorphism of $\tilde{\mathcal{H}}_{(\epsilon, 1)}^{\sharp}$ with $\Omega_{n}^{R}(\mathcal{H})$ as vector spaces (cf. (19)). Roughly speaking, under this isomorphism, the (inverse) Karoubi operator $\kappa^{\prime}$ and the cyclic permutation operator $\tau_{n}$ restricted to $\left(\tilde{\mathcal{H}}_{(\epsilon, 1)}^{\sharp}\right)_{n}$ coincide modulo the sign. Although in general $\tau_{n}\left(\left(\tilde{\mathcal{H}}_{(\epsilon, 1)}^{\sharp}\right)_{n}\right) \nsubseteq\left(\tilde{\mathcal{H}}_{(\epsilon, 1)}^{\sharp}\right)_{n}$, since $\sigma_{n}\left(\tau_{n}(\theta)\right) \neq 0, \theta \in\left(\tilde{\mathcal{H}}_{(\epsilon, 1)}^{\sharp}\right)_{n}$, for our purposes it is enough to consider only those elements $\theta$ whose image under $\tau_{n}$ belongs to $\left(\tilde{\mathcal{H}}_{(\epsilon, 1)}^{\sharp}\right)_{n}$. For instance, from the identities satisfied by the codegeneracies and cyclic operator, it follows that $\tau_{n-1} \circ \tilde{\sigma}_{0} \circ\left(1-\tau_{n}\right)(\theta) \in\left(\tilde{\mathcal{H}}_{(\epsilon, 1)}^{\sharp}\right)_{n-1}$ for all $\theta \in\left(\tilde{\mathcal{H}}_{(\epsilon, 1)}^{\sharp}\right)_{n}$. Still for all $\theta \in\left(\tilde{\mathcal{H}}_{(\epsilon, 1)}^{\sharp}\right)_{n}$, one can also note that $\left(\pi^{R}\right)^{\otimes}$ maps $\tau_{n}(\theta)$ to $\kappa^{\prime}\left(\left(\pi^{R}\right)^{\otimes}(\theta)\right)$, as follows from the formula (41) and the fact that $\pi^{R}(1)=0$. It is also easy to see that under this isomorphism the map $\tilde{\sigma}_{0} \circ\left(1-\tau_{n}\right)\left(\tilde{\sigma}_{0}\right.$ is defined in $\left.(45)\right)$ coincides with the Hochschild operator $b^{\prime}$. (Compare the formula (45) with (40) and observe that $\tilde{\sigma}_{0} \circ \tau_{n}=0$ on $\operatorname{ker} \sigma_{1}$.) One concludes (compare the signs) that under this map the operator $\tilde{B}^{\prime}$ on $\tilde{\mathcal{H}}_{(\epsilon, 1)}^{\sharp}$ corresponds to the operator $\left(\sum_{i=0}^{n-1}\left(\kappa^{\prime}\right)^{i}\right) b^{\prime}$ on the subspace $\Omega_{n}^{R}(\mathcal{H})$. It is also easy to see that under the same map the Hochschild differential $\tilde{b}^{\prime}$ on $\mathcal{H}_{(\epsilon, 1)}^{\sharp}$ corresponds to the "de Rham differential" $d$.

The only remaining problem is that the Hochschild and "de Rham" operators change their rôles under this isomorphism: $\tilde{b}^{\prime}$ corresponds to $d$, and $\tilde{\sigma}_{0} \circ\left(1-\tau_{n}\right)$ to $b^{\prime}$. The result of this confusion is that the operator $\tilde{B}^{\prime}$ on $\tilde{\mathcal{H}}_{(\epsilon, 1)}^{\sharp}$ corresponds to $\sum_{j=0}^{n-1}\left(\kappa^{\prime}\right)^{j} b^{\prime}$ and not to $B^{\prime}=\sum_{j=0}^{n}\left(\kappa^{\prime}\right)^{j} d$ that we considered above. (Note that the summation is taken over different sets of indices.) Therefore, unfortunately, the established isomorphism of spaces cannot be directly extended to the isomorphism of the associated periodic complexes. (In fact it is not even a map of mixed complexes.)

To cure this problem, recall $([8], \S 3)$ that the periodic homology super-complex associated with the mixed complex $(\Omega(\mathcal{H}), b, B)$ is quasi-isomorphic to the periodic supercomplex of its subcomplex $(P \Omega(\mathcal{H}), b, B)$, on which

$$
\begin{gathered}
(\kappa-1)^{2}=0, \\
\kappa=1-\frac{1}{n(n+1)} b B .
\end{gathered}
$$

Hence

$$
B=(n+1) d
$$

on $P \Omega_{n}(\mathcal{H})$. Here $P$ is the spectral projection associated to the generalized eigenvalue 1 of the operator $\kappa$. The chain homotopy is defined with the help of the Green operator $G$, which is equal to 0 on the image of $P$, and to the inverse of $1-\kappa$ on the complementary subcomplex, i.e. the image of $P^{\perp}=1-P$. Then $G$ commutes with the operators commuting with $\kappa$, for example with $d$ and $b$. Also, we have $[b+B, G d]=P^{\perp}$. 
These properties are a consequence of the spectral properties of the Karoubi operator $\kappa$. Clearly, similar statements are also true for the operators $\kappa^{\prime}, b^{\prime}, B^{\prime}$, see the formulae (37) and (38). By abuse of notation, we shall denote the corresponding spectral projection and the Green operator also by $P$ and $G$, respectively. It follows from (37) that the spectral decompositions associated with $\kappa$ and $\kappa^{\prime}$ just coincide, and hence the projections for these two Karoubi operators are identical.

Since the quasi-isomorphism $P$ and chain homotopy $G d$ (this pair is called a special deformation retraction in [8], §3) is expressed in terms of $\kappa^{\prime}, b^{\prime}$ and $d$ (see [8], p.392), which have been shown above to map $\Omega^{R}(\mathcal{H})$ to itself, we conclude that $\Omega^{R}(\mathcal{H})$ is quasiisomorphic to

$$
P \Omega^{R}(\mathcal{H}) \stackrel{\text { def }}{=} P \Omega(\mathcal{H}) \cap \Omega^{R}(\mathcal{H}) .
$$

The quasi-isomorphism and homotopy are given by the restrictions of $P$ and $G d$. In fact, it is not even necessary to use the cited formulas to show that $P$ and $G$ map $\Omega^{R}(\mathcal{H})$ to itself. To this end, it is enough to observe that this subcomplex is stable under the action of $\kappa^{\prime}$, whence one has a similar spectral decomposition and a set of projectors for $\Omega^{R}(\mathcal{H})$ as for $\Omega(\mathcal{H})$. Both maps $P$ and $G$ are defined only with the help of the spectral properties of $\kappa^{\prime}$.

Therefore, we see that the periodic cohomology of $\left(\Omega^{R}(\mathcal{H}), b^{\prime}, B^{\prime}\right)$ is equal to the cohomology of $\left(P \Omega^{R}(\mathcal{H}), b^{\prime}, \operatorname{deg} \circ d\right)$. Here deg is the operator which multiplies the degree $n$ homogeneous elements by $n$.

On the other hand, consider the map $G b^{\prime}$. It is easy to see that the pair $\left(P, G b^{\prime}\right)$ have all the properties of a special deformation retraction for $\left(\Omega^{R}(\mathcal{H}), d, \sum\left(\kappa^{\prime}\right)^{j} b^{\prime}\right)$. One just can repeat the reasoning from [8], p. 391 to prove this. (Recall that the operator $\sum\left(\kappa^{\prime}\right)^{j} b^{\prime}$ is the image of $\tilde{B}^{\prime}$ under the above isomorphism, which is why we consider it here.) Therefore, we conclude this time that the periodic cohomology of $\left(\Omega^{R}(\mathcal{H}), d, \sum\left(\kappa^{\prime}\right)^{j} b^{\prime}\right)$ equals the periodic cohomology of $P \Omega^{R}(\mathcal{H})$ with the induced differentials. From (48) it follows that $\sum\left(\kappa^{\prime}\right)^{j} b^{\prime}=b^{\prime} \circ \operatorname{deg}$ on $P \Omega^{R}(\mathcal{H})$.

Finally, the isomorphism of supercomplexes $C P_{1}(\mathcal{H})=\left(P \Omega_{\text {odd }}^{R}(\mathcal{H}) \oplus P \Omega_{\text {even }}^{R}(\mathcal{H}), b^{\prime}+\right.$ $\operatorname{deg} \circ d)$ and $C P_{2}(\mathcal{H})=\left(P \Omega_{\text {odd }}^{R}(\mathcal{H}) \oplus P \Omega_{\text {even }}^{R}(\mathcal{H}), d+b^{\prime} \circ \operatorname{deg}\right)$, where

$$
P \Omega_{\text {odd, even }}^{R}(\mathcal{H}):=\bigoplus_{i \equiv 1,0(\bmod 2)} P \Omega_{i}^{R}(\mathcal{H}),
$$

is established with the help of the following invertible chain operator:

$$
\begin{aligned}
L: C P_{1}(\mathcal{H}) & \rightarrow C P_{2}(\mathcal{H}), \\
L(\omega) & =\frac{1}{n !} \omega, \quad \omega \in P \Omega_{n}^{R}(\mathcal{H}) .
\end{aligned}
$$

In order to check that $\left(d+b^{\prime} \circ \operatorname{deg}\right) L=L\left(d+b^{\prime} \circ \operatorname{deg}\right)$, one computes:

$$
\begin{aligned}
\left(d+b^{\prime} \circ \operatorname{deg}\right) L(\omega) & =\left(d+b^{\prime} \circ \operatorname{deg}\right)\left(\frac{1}{n !} \omega\right)=\frac{1}{n !}\left(d \omega+n b^{\prime} \omega\right)=\frac{1}{n !} d \omega+\frac{1}{(n-1) !} b^{\prime} \omega \\
& =L((n+1) d \omega)+L\left(b^{\prime} \omega\right)=L\left(\left(b^{\prime}+\operatorname{deg} \circ d\right)(\omega)\right)
\end{aligned}
$$

for any $\omega \in P \Omega_{n}^{R}(\mathcal{H})$.

By a slight modification of this reasoning we obtain the following: 
Corollary 3. The periodic cohomology of the mixed complex $\Omega^{R}(\mathcal{H})$ (with differentials induced from $b$ and $B$ ) is isomorphic to the periodic Hopf-type cohomology of $\mathcal{H}$.

Proof. Just observe that the supercomplex $\left(\Omega^{R}(\mathcal{H}), b+B\right)$ is as before quasi-isomorphic to $\left(P \Omega^{R}(\mathcal{H}), \operatorname{deg} \circ d+b\right)$. (Recall that the spectral decomposition of $\kappa$ and $\kappa^{\prime}$ coincide.) Now, from (38) and (48) we conclude that at this subcomplex $b^{\prime}=b$.

Next, note that, since the space of left coinvariants in $\Omega(\mathcal{H})$ is also closed under the Hochschild boundary $b$ and the Karoubi operator $\kappa$, one can consider the corresponding mixed subcomplex and its periodic cohomology.

Proposition 4. Suppose that the conditions of Theorem 1 are satisfied. Then the antipode $S$ of the Hopf algebra $\mathcal{H}$ induces an isomorphism from the periodic complex of the mixed complex $\left(\Omega^{L}(\mathcal{H}), b, B\right)$ to the periodic complex of $\left(\Omega^{R}(\mathcal{H}), b^{\prime}, B^{\prime}\right)$.

Proof. Observe that, by virtue of the universal properties of $\Omega(\mathcal{H})$, the antipode $S$ can be extended to an anti-automorphism of $\Omega(\mathcal{H})$. Since we suppose that $S^{2}=1$ in $\mathcal{H}$, the same equation holds for this extension. Hence we get an involution anti-automorphism of the universal differential calculus of $\mathcal{H}$. Now, a straightforward computation shows that this map intertwines the right and left $\mathcal{H}$-comodule structures and differentials $b, \kappa$ and $b^{\prime}, \kappa^{\prime}$ in the mixed complex.

Corollary 5. Under the conditions of Theorem 1, the periodic cohomology of $\left(\Omega^{L}(\mathcal{H}), b, B\right)$ is canonically isomorphic to the periodic Hopf-type cohomology of $\mathcal{H}$.

REMARK 2. Note that the universality property of $\Omega(\mathcal{H})$ implies that all the maps defined at the level of $\mathcal{H}$ can be extended to this differential calculus. Thus, one can introduce the structure of a differential graded Hopf algebra on $\Omega(\mathcal{H})$. It would be an interesting problem to find out how much of this structure can be introduced on $\Omega^{R}(\mathcal{H})$ and on the corresponding cyclic cohomology.

3. General case: arbitrary $\delta$ and $\sigma$. In this section we shall investigate the case of a general modular pair in involution $(\delta, \sigma)$. To this end, we shall variate a little bit the construction we have just considered.

First recall that for any character $\xi$ of a Hopf algebra $\mathcal{H}$, one can introduce the following automorphism of $\mathcal{H}$ (called the "right convolution with the character $\xi$ "):

$$
\tilde{\xi}: \mathcal{H} \rightarrow \mathcal{H}, \quad \tilde{\xi}(a)=a \star \xi \stackrel{\text { def }}{=} a_{(1)} \xi\left(a_{(2)}\right) .
$$

The inverse of $\tilde{\xi}$ is given by the right convolution with $\xi^{-1} \stackrel{\text { def }}{=} \xi \circ S$.

The map $\tilde{\xi}$ is, in an evident way, a morphism of algebras, but it does not respect the coalgebra structure on $\mathcal{H}$. That is, $\Delta(\tilde{\xi}(a)) \neq \tilde{\xi}\left(a_{(1)}\right) \otimes \tilde{\xi}\left(a_{(2)}\right)$. In fact, in place of this equation one should use the following two:

$$
\begin{aligned}
& \Delta(\tilde{\xi}(a))=a_{(1)} \otimes \tilde{\xi}\left(a_{(2)}\right), \\
& \Delta(\tilde{\xi}(a))=\tilde{\xi}\left(a_{(1)}\right) \otimes A d_{\xi}\left(a_{(2)}\right),
\end{aligned}
$$

where

$$
A d_{\xi}(a)=\xi^{-1} \star a \star \xi=\xi\left(S\left(a_{(1)}\right)\right) a_{(2)} \xi\left(a_{(3)}\right) .
$$


It is easy to see that $A d$ defines an action of the group of characters of $\mathcal{H}$ on $\mathcal{H}$ by Hopf algebra homomorphisms. One calls it the adjoint action.

Since $\Omega(\mathcal{H})$ is the universal differential calculus, we conclude that the homomorphism $\tilde{\xi}$ can be extended to higher degree forms. By abuse of notation, we shall denote this map by the same symbol $\tilde{\xi}$. Observe that the equations (54) and (55) are fulfilled for this new map too, but in a slightly different form. One computes:

$$
\Delta_{R}(\tilde{\xi}(\omega))=(\mathrm{id} \otimes \tilde{\xi}) \Delta_{R}(\omega)=\left(\tilde{\xi} \otimes A d_{\xi}\right) \Delta_{R}(\omega), \quad \omega \in \Omega(\mathcal{H}) .
$$

On the other hand, $A d_{\xi}$ does not appear for the left coaction:

$$
\Delta_{L}(\tilde{\xi}(\omega))=(\operatorname{id} \otimes \tilde{\xi}) \Delta_{L}(\omega) .
$$

In fact, the formulae (57) and (58) are particular cases of the following observation. As remarked above, $\Omega(\mathcal{H})$ is a differential graded Hopf algebra. Its diagonal map will be denoted by $\tilde{\Delta}$. If once again, by abuse of notation, $A d_{\xi}$ denotes the automorphism of $\Omega(\mathcal{H})$ induced by the appropriate automorphism of $\mathcal{H}$, then the formulas (54) and (55) hold with $\tilde{\Delta}$ substituted for $\Delta$.

Note that, since $\tilde{\xi}$ is a map of differential graded algebras, one can use it to define a new differential structure on $\Omega(\mathcal{H})$. Just put

$$
d_{\xi}(\omega) \stackrel{\text { def }}{=} d(\tilde{\xi}(\omega))=\tilde{\xi}(d \omega)
$$

One easily checks the following statement, compare [8]:

Proposition 6. Let $\mathcal{H}$ be a Hopf algebra, $\xi$ its character and $d_{\xi}: \Omega_{*}(\mathcal{H}) \rightarrow \Omega_{*+1}(\mathcal{H})$ the map introduced above. Then:

(i) The map $d_{\xi}$ is a $\xi$-differential, i.e. it satisfies the following equations:

$$
\begin{aligned}
& d_{\xi}^{2}=0 \\
& d_{\xi}\left(\omega_{1} \omega_{2}\right)=d_{\xi}\left(\omega_{1}\right) \tilde{\xi}\left(\omega_{2}\right)+(-1)^{\left|\omega_{1}\right|} \tilde{\xi}\left(\omega_{1}\right) d_{\xi}\left(\omega_{2}\right) .
\end{aligned}
$$

(ii) The algebra $\Omega(\mathcal{H})$ equipped with the differential $d_{\xi}$ is the universal example of $\xi$-differential calculi on $\mathcal{H}$ in the sense that, for any algebra $\Omega$ satisfying

- $\Omega_{0}=\mathcal{H}$,

- $\Omega$ is equipped with a degree 1 map $d_{\Omega}, d_{\Omega}^{2}=0$, called a differential,

- $\tilde{\xi}$ extends to a degree 0 automorphism of $\Omega$ commuting with $d_{\Omega}$,

- its differential $d_{\Omega}$ satisfies (61),

there exists a unique homomorphism of differential graded algebras $\sharp_{\Omega}: \Omega(\mathcal{H}) \rightarrow \Omega$ such that $\left.\sharp_{\Omega}\right|_{\mathcal{H}}=\mathrm{id}_{\mathcal{H}}$.

(iii) Any element $\theta$ in $\Omega_{n}(\mathcal{H})$ can be represented in a unique way in the form

$$
\theta=\sum_{i} \tilde{\xi}\left(a_{0}^{i}\right) d_{\xi}\left(a_{1}^{i}\right) d_{\xi}\left(a_{2}^{i}\right) \ldots d_{\xi}\left(a_{n}^{i}\right)=\sum_{i} \tilde{a}_{0}^{i} d_{\xi}\left(a_{1}^{i}\right) d_{\xi}\left(a_{2}^{i}\right) \ldots d_{\xi}\left(a_{n}^{i}\right)
$$

for some $a_{i}^{\alpha} \in \mathcal{H}$ (here we put $\left.\tilde{a}=\tilde{\xi}(a)\right)$. Similarly, any element $\theta$ can be written in the form

$$
\theta=\sum_{j} d_{\xi}\left(b_{0}^{j}\right) d_{\xi}\left(b_{1}^{j}\right) \ldots d_{\xi}\left(b_{n-1}^{j}\right) \tilde{\xi}\left(b_{n}^{j}\right)=\sum_{j} d_{\xi}\left(b_{0}^{j}\right) d_{\xi}\left(b_{1}^{j}\right) \ldots d_{\xi}\left(b_{n-1}^{j}\right) \tilde{b}_{n}^{j}
$$


All this is checked by a straightforward inspection of definitions. Below we will denote the universal differential calculus $\Omega(\mathcal{H})$ with the differential $d_{\xi}$ by $\Omega_{\xi}(\mathcal{H})$. We shall also use the presentation of part (iii) to write the elements of $\Omega_{\xi}(\mathcal{H})$.

Now it is natural to write the left and right coactions of $\mathcal{H}$ on $\Omega(\mathcal{H})$ in terms of the formula (62). By the formulae (57) and (58), one gets

$$
\begin{aligned}
& \Delta_{R}\left(d_{\xi}(\omega)\right)=\left(d_{\xi} \otimes A d_{\xi}\right) \Delta_{R}(\omega), \\
& \Delta_{L}\left(d_{\xi}(\omega)\right)=\left(\operatorname{id} \otimes d_{\xi}\right) \Delta_{L}(\omega) .
\end{aligned}
$$

Hence, the formulae (17) and (18) become

$$
\begin{aligned}
& \Delta_{R}(\theta)=\sum_{i} \tilde{\xi}\left(a_{0,(1)}^{i}\right) d_{\xi} a_{1,(1)}^{i} d_{\xi} a_{2,(1)}^{i} \ldots d_{\xi} a_{n,(1)}^{i} \otimes A d_{\xi}\left(a_{0,(2)}^{i} a_{1,(2)}^{i} a_{2,(2)}^{i} \ldots a_{n,(2)}^{i}\right), \\
& \Delta_{L}(\theta)=\sum_{i} a_{0,(1)}^{i} a_{1,(1)}^{i} a_{2,(1)}^{i} \ldots a_{n,(1)}^{i} \otimes \tilde{\xi}\left(a_{0,(2)}^{i}\right) d_{\xi} a_{1,(2)}^{i} d_{\xi} a_{2,(2)}^{i} \ldots d_{\xi} a_{n,(2)}^{i},
\end{aligned}
$$

and similarly for the presentation (63):

$$
\begin{gathered}
\Delta_{R}(\theta)=\sum_{j} d_{\xi} b_{0,(1)}^{j} d_{\xi} b_{1,(2)}^{j} \ldots d_{\xi} b_{n-1,(1)}^{j} \tilde{\xi}\left(b_{n,(1)}^{j}\right) \otimes A d_{\xi}\left(b_{0,(2)}^{j} b_{1,(2)}^{j} \ldots b_{n-1,(2)}^{j} b_{n,(2)}^{j}\right), \\
\Delta_{L}(\theta)=\sum_{j} b_{0,(1)}^{j} b_{1,(1)}^{j} \ldots b_{n-1,(1)}^{j} b_{n,(1)}^{j} \otimes d_{\xi} b_{0,(2)}^{j} d_{\xi} b_{1,(2)}^{j} \ldots d_{\xi} b_{n-1,(2)}^{j} \tilde{\xi}\left(b_{n,(2)}^{j}\right) .
\end{gathered}
$$

Here we have used the fact that $A d_{\xi}$ is a Hopf algebra homomorphism.

To put the above considerations short, one can say that $\left(\Omega_{\xi}(\mathcal{H}), d_{\xi}\right)$ is the universal $\xi$-differential algebra consisting of linear combinations of elements of the form $\tilde{a}_{0} d_{\xi} a_{1} d_{\xi} a_{2} \ldots d_{\xi} a_{n}$ (or $d_{\xi} b_{0} d_{\xi} b_{1} \ldots d_{\xi} b_{n-1} \tilde{b}_{n}$ ), and on which the Hopf algebra $\mathcal{H}$ coacts on both sides by the formulae (66) and (67) (respectively by (68) and (69)). We shall use this notation below, though it is not absolutely necessary, since it is just another way to speak about the universal calculus $\Omega(\mathcal{H})$.

As before, one can consider the spaces of right and left coinvariants in $\Omega_{\xi}(\mathcal{H})$. For instance, the space of right ones, $\Omega_{\xi}^{R}(\mathcal{H})$, consists of the tensor powers of the space spanned by the elements

$$
\begin{aligned}
\pi^{R}(a) & =d\left(a_{(1)}\right) S\left(a_{(2)}\right) \\
& =d_{\xi}\left(a_{(1)}\right) \tilde{\xi}^{-1}\left(a_{(2)}\right) S\left(a_{(3)}\right) \\
& =d_{\xi}\left(a_{(1)}\right) S_{\xi^{-1}}\left(a_{(2)}\right), \quad a \in \operatorname{ker} \epsilon
\end{aligned}
$$

As before, $\Omega_{\xi}^{R}(\mathcal{H})$ is a differential graded subalgebra of $\Omega_{\xi}(\mathcal{H})$. This follows directly from (66) (see also (64)). In addition to the usual coinvariants, one can consider the space of elements $\theta$ such that

$$
\Delta_{R}(\theta)=\theta \otimes \sigma
$$

for some group-like element $\sigma$. We shall call such elements (right) $\sigma$-coinvariants. Let $\Omega_{\sigma}^{R}(\mathcal{H})$ (respectively $\Omega_{\xi, \sigma}^{R}(\mathcal{H})$ ) denote the space of right $\sigma$-coinvariants in $\Omega(\mathcal{H}$ ) (resp. in $\Omega_{\xi}(\mathcal{H})$ ). Clearly, since $\Delta_{R}$ commutes with the differential $d, \Omega_{\sigma}^{R}(\mathcal{H})$ is a differential graded subalgebra of $\Omega(\mathcal{H})$. A similar statement holds for $d_{\xi}, \Omega_{\xi, \sigma}^{R}(\mathcal{H})$ and $\Omega_{\xi}(\mathcal{H})$.

Proposition 7. The differential $d_{\xi}$ maps the space of $\sigma$-coinvariants into itself. Moreover, the space $\Omega_{\xi, \sigma}^{R}(\mathcal{H})$ is a differential graded $\Omega_{\xi}^{R}(\mathcal{H})$-sub-bimodule of $\Omega_{\xi}(\mathcal{H})$. 
Proof. Note that the right multiplication by $\sigma$ establishes an isomorphism between the space of (right) coinvariants and the space of (right) $\sigma$-coinvariants. The inverse is given by the multiplication by $\sigma^{-1}=S(\sigma)$. Hence, any element in $\Omega_{\xi, \sigma}^{R}(\mathcal{H})$ is representable in the form

$$
\theta=\theta^{\prime} \cdot \sigma
$$

for a suitable $\theta^{\prime} \in \Omega_{\xi}^{R}(\mathcal{H})$. Hence, it is enough to show that $d_{\xi}(\sigma) \in \Omega_{\xi, \sigma}^{R}(\mathcal{H})$. We compute:

$$
d_{\xi}(\sigma)=d(\tilde{\xi}(\sigma))=d(\sigma \xi(\sigma))
$$

which is, clearly, (right) $\sigma$-coinvariant, since $d$ commutes with the coaction. Here we have used the fact that $\sigma$ is group-like, i.e. $\Delta(\sigma)=\sigma \otimes \sigma$.

Finally, the fact that $\Omega_{\xi, \sigma}^{R}(\mathcal{H})=\Omega_{\sigma}^{R}(\mathcal{H})$ is a left $\Omega_{\xi}^{R}(\mathcal{H})$-module is a consequence of the presentation (72). Since the left multiplication by $\sigma^{ \pm 1}$ also establishes an isomorphism between $\Omega_{\xi, \sigma}^{R}(\mathcal{H})$ and $\Omega_{\xi}^{R}(\mathcal{H})$, the conclusion follows.

Let us now define the $\xi$-twisted cyclic structure on $\Omega_{\xi}(\mathcal{H})=\Omega(\mathcal{H})$ by giving analogs of the Hochschild operator $b$ (or $b^{\prime}$ ) and Karoubi operator $\kappa$ (or $\kappa^{\prime}$ ). In other words, let us use the presentations $(62)$ and $(63)$ to define the following operators on $\Omega(\mathcal{H})$. Put (compare (32)-(36))

$$
\begin{aligned}
b_{\xi}\left(\omega d_{\xi} a\right) & =(-1)^{|\omega|}(\omega \tilde{\xi}(a)-a \omega), \\
\kappa_{\xi} & =1-b_{\xi} d-d b_{\xi},
\end{aligned}
$$

or explicitly

$$
\kappa_{\xi}\left(\omega d_{\xi} a\right)=(-1)^{|\omega|} d a \omega
$$

Similarly, define

$$
\begin{aligned}
b_{\xi}^{\prime}\left(d_{\xi} a \omega\right) & =\tilde{\xi}(a) \omega-\omega a, \\
\kappa_{\xi}^{\prime}\left(d_{\xi} a \omega\right) & =(-1)^{|\omega|} \omega d a .
\end{aligned}
$$

It is clear that the operators $b_{\xi}$ and $b_{\xi}^{\prime}$ are well defined, since $\tilde{\xi}(1)=1$. Also, observe that

$$
\begin{aligned}
\kappa_{\xi}^{\prime} & =\kappa_{\xi^{-1}}^{-1}, \\
b_{\xi}^{\prime} & =-b_{\xi^{-1}} \kappa_{\xi}^{\prime} .
\end{aligned}
$$

Once again, one easily checks that these operators have all the properties of the standard ones, listed in [8], $\S 3$, with few modifications that are due to the $\tilde{\xi}$-twist of the structure. In fact, the following proposition holds (compare [8], §3).

Proposition 8. Let $\mathcal{H}$ be a Hopf algebra, $\xi$ its character, $b_{\xi}, b_{\xi}^{\prime}, \kappa_{\xi}$ and $\kappa_{\xi}^{\prime}$ be the operators on $\Omega(\mathcal{H})$, introduced in (74)-(78), $d$ and $d_{\xi}$ the standard and twisted differentials, respectively, on $\Omega(\mathcal{H})$ (see (59)), and $\tilde{\xi}$ the automorphism of $\Omega(\mathcal{H})$ defined by the right convolution with the character $\xi$ on $\mathcal{H}$. Then:

(i) $b_{\xi}^{2}=\left(b_{\xi}^{\prime}\right)^{2}=0$.

(ii) The operators $b_{\xi}, \kappa_{\xi}, d, d_{\xi}, \tilde{\xi}$ (similarly $b_{\xi}^{\prime}, \kappa_{\xi}^{\prime}$ ) commute in the following way:

$$
\begin{array}{r}
{\left[b_{\xi}, \kappa_{\xi}\right]=\left[d, \kappa_{\xi}\right]=\left[d_{\xi}, \kappa_{\xi}\right]=0,} \\
{\left[\tilde{\xi}, \kappa_{\xi}\right]=\left[\tilde{\xi}, b_{\xi}\right]=0 .}
\end{array}
$$


The following identities hold on the homogeneous elements of degree $n$ in $\Omega_{\xi}(\mathcal{H})$ :

(iii) $\kappa_{\xi}^{n+1} d_{\xi}=\tilde{\xi}^{-1} d_{\xi}=d$

(iv) $\kappa_{\xi}^{n}=\tilde{\xi}^{-1}+b_{\xi} \kappa_{\xi}^{n} d$.

(v) $\kappa_{\xi}^{n} b_{\xi}=\tilde{\xi}^{-1} b_{\xi}$.

(vi) $\kappa_{\xi}^{n+1}=\tilde{\xi}^{-1}\left(1-d b_{\xi}\right)$.

(vii) $\left(\kappa_{\xi}^{n}-\tilde{\xi}^{-1}\right)\left(\kappa_{\xi}^{n+1}-\tilde{\xi}^{-1}\right)=0$.

(viii) Let

$$
B_{\xi}=\sum_{j=0}^{n} \kappa_{\xi}^{j} d .
$$

Then $B_{\xi} d=d B_{\xi}=B_{\xi}^{2}=0$.

(ix) On the set of fixed points of the operator $\tilde{\xi}$ one has

$$
\kappa_{\xi}^{n(n+1)}-1=b_{\xi} B_{\xi}=-B_{\xi} b_{\xi} .
$$

Similar equations hold for the primed operators. (The version of the operator $B_{\xi}$ constructed with the help of the primed Hochschild and Karoubi operators will be denoted $B_{\xi}^{\prime}$.)

Proof. Part (i) is checked by a direct inspection of formulas. Part (ii) follows from part (i), (75) and the fact that $\tilde{\xi}$ is a differential graded algebra automorphism, so that it commutes with $b_{\xi}$ and $d_{\xi}$, and, consequently, also with $\kappa_{\xi}$. All the rest is obtained by mimicking the reasoning of the cited paper and taking into consideration the fact that $\tilde{\xi}$ commutes with all the operators introduced above. For instance, let us prove part (iv). We compute, using the presentation (62), the formula (76), $d_{\xi}=d \tilde{\xi}$ and the definitions of $b_{\xi}$ and $d_{\xi}$ :

$$
\begin{aligned}
\kappa_{\xi}^{n}\left(\tilde{a}_{0} d_{\xi} a_{1} \ldots d_{\xi} a_{n}\right) & =d a_{1} \ldots d a_{n} \tilde{a}_{0}=\tilde{\xi}^{-1}\left(d_{\xi} a_{1} \ldots d_{\xi} a_{n} \tilde{\xi}\left(\tilde{a}_{0}\right)\right) \\
& =\tilde{\xi}^{-1}\left(\tilde{a}_{0} d_{\xi} a_{1} \ldots d_{\xi} a_{n}+(-1)^{n} b_{\xi}\left(d_{\xi} a_{1} \ldots d_{\xi} a_{n} d_{\xi} \tilde{a}_{0}\right)\right) \\
& =\tilde{\xi}^{-1}\left(\tilde{a}_{0} d_{\xi} a_{1} \ldots d_{\xi} a_{n}+(-1)^{n} b_{\xi} \kappa_{\xi}^{n}\left(d_{\xi} \tilde{a}_{0} d_{\xi} \tilde{\xi}\left(a_{1}\right) \ldots d_{\xi} \tilde{\xi}\left(a_{n}\right)\right)\right) \\
& =\tilde{\xi}^{-1}\left(\tilde{a}_{0} d_{\xi} a_{1} \ldots d_{\xi} a_{n}+b_{\xi} \kappa_{\xi}^{n} d_{\xi}\left(\tilde{a}_{0} d_{\xi} a_{1} \ldots d_{\xi} a_{n}\right)\right) \\
& =\left(\tilde{\xi}^{-1}+b_{\xi} \kappa_{\xi}^{n} d\right)\left(\tilde{a}_{0} d_{\xi} a_{1} \ldots d_{\xi} a_{n}\right) .
\end{aligned}
$$

In the case of the primed operators the proof is completely similar.

Now we come to the main result of this paper. The following theorem is a straightforward generalization of Theorem 1 of Section 2 .

TheOREM 9. Let $(\delta, \sigma)$ be a modular pair in involution, and let $\xi=\delta^{-1}$ be the convolution inverse of $\delta$. Then the space $\Omega_{\xi, \sigma}^{R}(\mathcal{H})$ of $\sigma$-coinvariants in $\Omega_{\xi}(\mathcal{H})$ is stable under the twisted Hochschild $\left(b_{\xi}^{\prime}\right.$ and $\left.b_{\delta}\right)$ and Karoubi $\left(\kappa_{\xi}^{\prime}\right.$ and $\kappa_{\delta}=\left(\kappa_{\xi}^{\prime}\right)^{-1}$, see (79)) operators. Moreover, $\Omega_{\xi, \sigma}^{R}(\mathcal{H})$ is fixed under the operator $\tilde{\xi}$, and the associated periodic cohomology of the primed mixed complex (i.e. the complex with operators $b_{\xi}^{\prime}$ and $B_{\xi}^{\prime}$ ) is naturally isomorphic to the Hopf-type periodic cohomology $H P_{\delta, \sigma}(\mathcal{H})$ of A. Connes and H. Moscovici.

Proof. It is obtained in a way absolutely similar to the proof of Theorem 1 . First of all, we establish the first part of this statement. Once again, we prefer to work with the 
primed versions of cyclic operators - the statement about their unprimed counterparts is a direct consequence of the equations (79) and (80).

Recall that $\xi=\delta^{-1}$ and check that $b_{\xi}^{\prime}(\omega) \in \Omega_{\sigma}(\mathcal{H})$ for all $\omega \in \Omega_{\sigma}(\mathcal{H})($ compare $(40))$ :

$$
\begin{aligned}
b_{\xi}^{\prime}(\omega) & =b_{\xi}^{\prime}\left(\pi^{R}\left(a_{1}\right) \ldots \pi^{R}\left(a_{n}\right) \sigma\right) \\
& =b_{\xi}^{\prime}\left(d_{\xi}\left(a_{1,(1)}\right) S_{\delta}\left(a_{1,(2)}\right) \omega^{\prime} \sigma\right)=\tilde{\xi}\left(a_{1,(1)}\right) S_{\delta}\left(a_{1,(2)}\right) \omega^{\prime} \sigma-S_{\delta}\left(a_{1,(2)}\right) \omega^{\prime} \sigma a_{1,(1)} \\
& =-\left(\pi^{R}\left(S\left(a_{1,(n+1)}\right) a_{2}\right) \ldots \pi^{R}\left(S\left(a_{1,(3)}\right) a_{n}\right)\right)\left(S_{\delta}\left(a_{1,(2)}\right) \sigma a_{1,(1)}\right) \\
& =-\left(\pi^{R}\left(S\left(a_{1,(n+1)}\right) a_{2}\right) \ldots \pi^{R}\left(S\left(a_{1,(3)}\right) a_{n}\right)\right)\left(S_{\delta}\left(a_{1,(2)}\right) S_{\delta}^{2}\left(a_{1,(1)}\right)\right) \sigma \\
& =-\left(\pi^{R}\left(S\left(a_{1,(n+1)}\right) a_{2}\right) \ldots \pi^{R}\left(S\left(a_{1,(3)}\right) a_{n}\right)\right)\left(S_{\delta}\left(S_{\delta}\left(a_{1,(1)}\right) a_{1,(2)}\right)\right) \sigma \\
& =-\pi^{R}\left(S\left(a_{1,(n-1)}\right) a_{2}\right) \pi^{R}\left(S\left(a_{1,(n-2)}\right) a_{3}\right) \ldots \pi^{R}\left(S_{\delta}\left(a_{1,(1)}\right) a_{n}\right) \sigma .
\end{aligned}
$$

We have used the fact that $S_{\delta}^{2}(a)=\sigma a \sigma^{-1}$, and the following properties of $S_{\delta}$ :

$$
\begin{aligned}
S_{\delta}(a b) & =S_{\delta}(b) S_{\delta}(a), \\
\Delta\left(S_{\delta}(a)\right) & =S\left(a_{(1)}\right) \otimes S_{\delta}\left(a_{(2)}\right), \\
S_{\delta}\left(a_{(1)}\right) a_{(2)} & =\delta(a) .
\end{aligned}
$$

All this is proven by direct computations (see, e.g., [6]).

Similarly to the observation following the equation (40), one concludes that $\kappa_{\xi}^{\prime}$ maps $\Omega_{\sigma}(\mathcal{H})$ to itself by a mere inspection of definitions. But we prefer to give an explicit proof here too. We compute (cf. (41)):

$$
\begin{aligned}
& \kappa_{\xi}^{\prime}\left(d_{\xi} a_{1,(1)} S_{\delta}\left(a_{1,(2)}\right) \omega^{\prime} \sigma\right)=(-1)^{\left|\omega^{\prime}\right|} S_{\delta}\left(a_{1,(2)}\right) \omega^{\prime} \sigma d a_{1,(1)} \\
& =(-1)^{\left|\omega^{\prime}\right|}\left(\pi^{R}\left(S\left(a_{1,(n+1)}\right) a_{2}\right) \ldots \pi^{R}\left(S\left(a_{1,(3)}\right) a_{n}\right)\right)\left(S_{\delta}\left(a_{1,(2)}\right) \sigma d a_{1,(1)}\right) .
\end{aligned}
$$

Now, consider the last term of this expression separately (we omit the subscript 1 for brevity):

$$
\begin{aligned}
S_{\delta}\left(a_{(2)}\right) \sigma d a_{(1)} & =S_{\delta}\left(a_{(3)}\right) \sigma \pi^{R}\left(a_{(1)}\right) a_{(2)} \\
& =\pi^{R}\left(S\left(a_{(4)}\right) \sigma a_{(1)}-S\left(a_{(4)}\right) \sigma \epsilon\left(a_{(1)}\right)\right) S_{\delta}\left(a_{(3)}\right) \sigma a_{(2)} \\
& =\pi^{R}\left(S\left(a_{(4)}\right) \sigma a_{(1)}\right) S_{\delta}\left(a_{(3)}\right) S_{\delta}^{2}\left(a_{(2)}\right) \sigma-\pi^{R}\left(S\left(a_{(3)}\right) \sigma\right) S_{\delta}\left(a_{(2)}\right) S_{\delta}^{2}\left(a_{(1)}\right) \sigma \\
& =\pi^{R}\left(S\left(a_{(4)}\right) \sigma a_{(1)}\right) S_{\delta}\left(S_{\delta}\left(a_{(2)}\right) a_{(3)}\right) \sigma-\pi^{R}\left(S\left(a_{(3)}\right) \sigma\right) S_{\delta}\left(S_{\delta}\left(a_{(1)}\right) a_{(2)}\right) \sigma \\
& =\pi^{R}\left(\delta\left(a_{(2)}\right) S\left(a_{(3)}\right) \sigma a_{(1)}\right) \sigma-\pi^{R}\left(\delta\left(a_{(1)}\right) S\left(a_{(2)}\right) \sigma\right) \sigma \\
& =\pi^{R}\left(S_{\delta}\left(a_{(2)}\right) S_{\delta}^{2}\left(a_{(1)}\right) \sigma\right) \sigma-\pi^{R}\left(S_{\delta}(a) \sigma\right) \sigma \\
& =-\pi^{R}\left(\left(S_{\delta}(a)-\delta(a)\right) \sigma\right) \sigma .
\end{aligned}
$$

Here we used the equation (24).

Now, the equations (87) and (88) show that, identifying $\left(\Omega_{\sigma}^{R}\right)_{n}(\mathcal{H})$ with $(\operatorname{ker} \epsilon)^{\otimes n}$ (see Proposition 7$)$, one can write the twisted Karoubi operator $\kappa_{\xi}^{\prime}$ as follows:

$$
\kappa_{\xi}^{\prime}\left(h_{1}, h_{2}, \ldots, h_{n}\right)=\operatorname{proj}^{\prime \prime} S_{\delta}\left(h_{1}\right)\left(h_{2}, \ldots, h_{n}, \sigma\right),
$$

where proj" $^{\prime \prime}$ is the following projection:

$$
\operatorname{proj}^{\prime \prime}\left(h_{1}, h_{2}, \ldots, h_{n}\right)=\left(h_{1}-\epsilon\left(h_{1}\right) \cdot 1, h_{2}-\epsilon\left(h_{2}\right) \cdot 1, \ldots, h_{n}-\epsilon\left(h_{n}\right) \sigma\right) .
$$

Finally, $\tilde{\xi}$ acts trivially on $\Omega_{\sigma}^{R}(\mathcal{H})$, since $\delta(\sigma)=1, \xi=\delta^{-1}$, and part (ix) of Proposition 8 is fulfilled. The rest of the proof reproduces the reasoning at the end of the proof of 
Theorem 1 of Section 2 with minor technical changes. For instance, the homomorphism (46) should be changed to

$$
\mathcal{H}^{\otimes n} \ni h_{1} \otimes h_{2} \otimes \ldots \otimes h_{n} \mapsto-\pi^{R}\left(h_{1}\right) \ldots \pi^{R}\left(h_{n}\right) \sigma \in \Omega_{n}^{R}(\mathcal{H}) .
$$

Similarly to Corollary 3 , we obtain:

Corollary 10. The periodic cyclic cohomology of the complex $\left(\Omega_{\sigma}^{R}(\mathcal{H}), b_{\delta}, B_{\delta}\right)$ is isomorphic to the Connes-Moscovici periodic cyclic cohomology $H_{\delta, \sigma}(\mathcal{H})$.

Now, similarly to the discussion at the end of Section 2, one can try to obtain an analog of Theorem 9 for the space of left $\sigma$-coinvariants. This is a good exercise and is left to the reader.

4. Conclusions. In this section, we shall make a few remarks concerning the possible ways to generalize the Hopf-type cohomology. First of all, consider the special case discussed in Section 2 (i.e. we suppose that $S^{2}=$ id and consider the modular pair $\epsilon, 1$ ). Since both $\Omega^{R}(\mathcal{H})$ and $\Omega^{L}(\mathcal{H})$ are closed under the mixed complex differentials of $\Omega(\mathcal{H})$, we conclude that the subspace of bi-invariants is also a sub-mixed complex in $\Omega(\mathcal{H})$. Moreover, this subcomplex is stable under the involution $S$. The corresponding periodic and dihedral periodic homology will be denoted by $H P_{b i, \epsilon, 1}(\mathcal{H})$ and $H D_{\epsilon, 1}(\mathcal{H})$, respectively.

In the case of an arbitrary modular pair in involution $\delta, \sigma$, the same construction allows one to define an analogue of bi-invariant cohomology. If $\sigma=1$, one can reproduce the dihedral construction too. What is the analog of dihedral cohomology in the case of arbitrary $\sigma$ is not so evident.

Note that if the Hopf algebra $\mathcal{H}$ is cocommutative, the spaces of left and right (co)invariants coincide, so we see that in this case bi-invariant cohomology is isomorphic to the Hopf-type one. In general the answer is not clear. Besides this, it isn't clear whether it is possible to define this type of bi-invariant and dihedral cohomology in an $\Omega(\mathcal{H})$-independent way.

Another important observation is that in order to define the twisted cyclic structure on $\Omega(\mathcal{H})$ (which is equivalent, up to a change of basis, to $\Omega_{\xi}(\mathcal{H})$ ), we didn't really use the fact that the isomorphism $\tilde{\xi}$ was the convolution with a character of $\mathcal{H}$, nor even did we use the fact that $\mathcal{H}$ is a Hopf algebra. One can go along the very same way for any automorphism $f$ of any algebra $\mathcal{A}$ to define $f$-twisted cyclic operators on its universal differential calculus $\Omega(\mathcal{A})$. One can denote the corresponding cyclic (respectively negative cyclic, periodic cyclic, etc.) homology by $H C_{f}(\mathcal{A})\left(\operatorname{resp} . H C_{f}^{-}(\mathcal{A}), H P_{f}(\mathcal{A})\right.$, etc.). For example, one can take an automorphism

$$
f: \mathcal{H} \rightarrow \mathcal{H}, \quad f(a)=\alpha \star a \star \beta
$$

( $\alpha$ and $\beta$ are characters of $\mathcal{H}$ ). Then, if $\left(\sigma S_{\alpha, \beta}\right)^{2}=1$, passing to $\sigma$-coinvariants one obtains the construction of [23]. ( $S_{\alpha, \beta}$ is the evident generalization of the map $S_{\delta}$.)

In fact, the homology $H C_{f}(\mathcal{A})$ can be defined in a quite $\Omega(\mathcal{A})$-independent way, see for example [19]. Thus one can define the $f$-twisted cyclic module as follows: $C C_{n}(\mathcal{A}, f)=$ $\mathcal{A}^{\otimes n+1}$ and

$$
\delta_{i}^{f}\left(h_{0}, h_{1}, \ldots, h_{n}\right)= \begin{cases}\left(h_{0}, \ldots, h_{i} h_{i+1}, \ldots, h_{n}\right), & i=0, \ldots, n-1 \\ \left(f\left(h_{n}\right) h_{0}, \ldots, h_{n-1}\right), & i=n\end{cases}
$$




$$
\begin{aligned}
& \sigma_{i}^{f}\left(h_{0}, h_{1}, \ldots, h_{n}\right)=\left(h_{0}, \ldots, h_{i-1}, 1, h_{i}, \ldots, h_{n}\right), \quad 1 \leq i \leq n, \\
& \tau_{n}^{f}\left(h_{0}, h_{1}, \ldots, h_{n}\right)=(-1)^{n}\left(f\left(h_{n}\right), h_{0}, \ldots, h_{n}\right) .
\end{aligned}
$$

Then all the usual equations of the cyclic operations are fulfilled for these ones, except that one should substitute an appropriate tensor power of $f$ for the identity operator in certain formulas. Furthermore, considering the corresponding spaces of fixed points, one defines the twisted homology theories in the usual way, by means of the cyclic double complex.

One more way to generalize the constructions above is to use the remark at the end of Section 2. Taking into consideration the fact that $\Omega(\mathcal{H})$ is a (differential graded) Hopf algebra, one can consider it as an input to the Connes-Moscovici construction. Then the universality property of $\Omega(\mathcal{H})$ guarantees that $S_{\delta}$ extends to a homomorphism of this algebra in such a way that all the properties of this map are valid for the extension too. What one obtains in this way is a construction very similar to the non-commutative Weil complex of Crainic [6]. On the other hand, a very similar construction was introduced by Đurđevič in the guise of universal characteristic classes for Hopf-Galois extensions. This matter will be a subject of thorough discussion in a forthcoming paper.

Finally, there are two more possible approaches to generalizing constructions presented in this paper. The first one consists in considering, instead of the subcomodule $\Omega_{\sigma}^{R}(\mathcal{H})$ of $\Omega(\mathcal{H})$ determined by a modular pair, an arbitrary $\mathcal{H}$-subcomodule of the same algebra which is stable under the Hochschild and "de Rham" operators $b$ and $d$ (equivalently, one can consider their primed analogs). Here one can plug in both the standard and the $\xi$-twisted versions of these operators. For instance, if $\delta=\epsilon$, such stable subcomodules are in one-one correspondence with all subcoalgebras $\mathcal{H}^{\prime}$ of $\mathcal{H}$, for which

$$
S\left(a_{(2)}\right) \mathcal{H}^{\prime} a_{(1)} \subseteq \mathcal{H}^{\prime}
$$

for all $a \in \mathcal{H}$ (cf. the isomorphisms (19), (21) and the formulas (40), (41)). If $\mathcal{H}$ is commutative, this is always true, and if $\mathcal{H}$ is cocommutative, this is equivalent to saying that $\mathcal{H}^{\prime}$ is stable under the adjoint action of $\mathcal{H}$ on itself. ${ }^{2}$

The second construction seems to be even more general. It is based on the following idea. It is a well-known fact (see [17]) that one can obtain a good variant of cyclic-type homology, called the non-commutative de Rham homology from the universal differential calculus $\Omega(\mathcal{A})$ of an algebra $\mathcal{A}$ simply by passing to the quotient complex

$$
\bar{\Omega}(\mathcal{A}) \stackrel{\text { def }}{=} \Omega(\mathcal{A}) /[\Omega(\mathcal{A}), \Omega(\mathcal{A})]
$$

where $[\Omega(\mathcal{A}), \Omega(\mathcal{A})]$ is the subspace of graded commutators of elements of $\Omega(\mathcal{A})$. One easily checks that the differential $d$ of $\Omega(\mathcal{A})$ descends to a differential in $\bar{\Omega}(\mathcal{A})$. A less trivial fact is that the induced homology of $\bar{\Omega}(\mathcal{A})$ coincides with a certain subspace of the cyclic homology of $\mathcal{A}$ (see the paper of Karoubi [17]).

Now, if we pass to the barred complex in the case of a Hopf algebra $\mathcal{H}$, we can no more say that $\mathcal{H}$ acts on it. In fact, this is not the case, unless $\mathcal{H}$ is commutative. But it is easy to see that the space of commutators $[\mathcal{H}, \mathcal{H}]$ is a coideal in $\mathcal{H}$, whence one can

\footnotetext{
${ }^{2}$ This condition resembles very much Schneider's normality condition of a Hopf subalgebra $[21]$.
} 
substitute the coalgebra $\overline{\mathcal{H}}=\mathcal{H} /[\mathcal{H}, \mathcal{H}]$ for $\mathcal{H}$. Then $\overline{\mathcal{H}}$ coacts on $\bar{\Omega}(\mathcal{H})$ on the right (as well as on the left) and it is possible to consider the space of coinvariants of this coaction, namely the space of those elements $\bar{\omega} \in \bar{\Omega}(\mathcal{H})$ that are sent to $\bar{\omega} \otimes \overline{1}$, where $\overline{1}$ is the group-like element in $\overline{\mathcal{H}}$ determined by $1 \in \mathcal{H}$.

This construction seems to play an important role in the theory of characteristic classes for Hopf-Galois extensions (see e.g. [10, 11]). On the other hand, an important application of the Hopf-type cohomology is the theory of characteristic classes of a Hopfmodule algebra. Since to any Hopf-module algebra one can associate its smashed product with $\mathcal{H}$, which is an example of a Hopf-Galois extension of an algebra, it seems interesting to investigate the relationship between the Connes and Moscovici construction of characteristic classes of a Hopf-module algebra and various constructions of characteristic classes for Hopf-Galois extensions (see the just quoted papers for definitions).

Acknowledgements. The author thanks the organizers of the school/conference Noncommutative Geometry and Quantum Groups for their hospitality, and Dr. P. M. Hajac for many valuable suggestions concerning the contents and presentation of this paper.

\section{References}

[1] R. Akbarpour and M. Khalkhali, Equivariant cyclic cohomology of Hopf module algebras, J. Reine Angew. Math. 559 (2003), 137-152.

[2] T. Brzeziński and S. Majid, Quantum group gauge theory on quantum spaces, Commun. Math. Phys. 157 (1993), 591-638. Erratum Comm. Math. Phys. 167 (1995) 235.

[3] A. Connes and H. Moscovici, Hopf algebras, cyclic cohomology and the transverse index theorem, Commun. Math. Phys. 198 (1998), 199-246.

[4] A. Connes and H. Moscovici, Cyclic cohomology and Hopf algebras, Letters Math. Phys. 48 (1999), 97-108.

[5] A. Connes and H. Moscovici, Cyclic cohomology and Hopf algebra symmetry, Lett. Math. Phys. 52 (2000), 1-28.

[6] M. Crainic, Cyclic cohomology of Hopf algebras, J. Pure Appl. Algebra 166 (2002), 29-66.

[7] J. Cuntz and D. Quillen, Algebra extensions and nonsingularity, J. Amer. Math. Soc. 8 (1995), 251-289.

[8] J. Cuntz and D. Quillen, Cyclic homology and nonsingularity, J. Amer. Math. Soc. 8 (1995), 373-442.

[9] M. Đurđevič, Geometry of quantum principal bundles I, Commun. Math. Phys. 175 (1996), 457-520.

[10] M. Đurđevič, Geometry of quantum principal bundles II, Rev. Math. Phys. 9 (5) (1997), 531-603.

[11] M. Đurđevič, Quantum principal bundles and their characteristic classes, Banach Center Publications 40 (1997), 303-313.

[12] P. M. Hajac, Strong connections on quantum principal bundles, Commun. Math. Phys. 182 (1996), 579-617.

[13] P. M. Hajac, M. Khalkhali, B. Rangipour and Y. Sommerhäuser, Hopf-cyclic homology and cohomology with coefficients, preprint: math.KT/0306288. 
[14] P. Jara and D. Stefan, Cyclic homology of Hopf-Galois extensions and Hopf algebras, preprint: math.KT/0307099.

[15] M. Khalkhali and B. Rangipour, A new cyclic module for Hopf algebras, K-Theory 27 (2002), 111-131.

[16] M. Khalkhali and B. Rangipour, Invariant cyclic homology, K-theory 28 (2003).

[17] M. Karoubi, Homologie cyclique et K-théorie, Astérisque 149 (1987).

[18] H. F. Kreimer and M. Takeuchi, Hopf algebras and Galois extensions of an algebra, Indiana Univ. Math. J. 30 (1981), 675-692.

[19] J. Kustermans, G. J. Murphy and L. Tuset, Differential calculi over quantum groups and twisted cyclic cocicles, J. Geom. Phys. 44 (2003), 570-594.

[20] J.-L. Loday, Cyclic Homology, Springer, Berlin, 1992.

[21] H. J. Schneider, Some remarks on exact sequences of quantum groups, Comm. Alg. 21 (1993), 3337-3357.

[22] M. E. Sweedler, Hopf Algebras, W. A. Benjamin, New York, 1969.

[23] R. Taillefer, Cyclic homology of Hopf algebras, K-Theory 24 (2001), 69-85.

[24] S. L. Woronowicz, Differential calculus on compact matrix pseudogroups (quantum groups), Commun. Math. Phys. 122 (1989), 125-170. 